\title{
Analysis on Composition and Antioxidative Properties of Phlorotannins Isolated from Japanese Eisenia and Ecklonia Species
}

\author{
Toshiyuki Shibata1,2*, Kohki Nagayama2,3, Shingo Sugiura1,2, Saki Makino1,2, \\ Mitsuyoshi Ueda',4, Yutaka Tamaru1 \\ ${ }^{1}$ Graduate School of Bioresources, Mie University, Mie, Japan \\ 2Japan Science and Technology Agency, Core Research for Evolutional Science and Technology, Tokyo, Japan \\ ${ }^{3}$ Kumamoto Prefectural Fisheries Research Center, Kumamoto, Japan \\ ${ }^{4}$ Graduate School of Agriculture, Kyoto University, Kyoto, Japan \\ Email: *shibata@bio.mie-u.ac.jp
}

Received 7 May 2015; accepted 27 September 2015; published 30 September 2015

Copyright (C) 2015 by authors and Scientific Research Publishing Inc.

This work is licensed under the Creative Commons Attribution-NonCommercial International License (CC BY-NC).

http://creativecommons.org/licenses/by/4.0/

(c) (i) Open Access

\section{Abstract}

The composition and antioxidative properties of crude phlorotannins isolated from Japanese Eisenia and Ecklonia species including cultured E. kurome were analyzed. As a result of liquid chromatography mass spectrometry analysis, it was suggested that the phlorotannins were composed of compounds containing 3 - 7 phloroglucinol units for Eisenia species and 3 - 8 phloroglucinol units for Ecklonia species. The antioxidant properties of phlorotannins were evaluated using the hydrophilic oxygen radical absorbance capacity (H-ORAC) assay, the measurements of superoxide anion radical scavenging activity (SOSA) with electron spin resonance method, and the 2,2-diphenyl-1-picrylhydrazyl (DPPH) radical scavenging activity and reducing power assay. It was clarified that the phlorotannins contained H-ORAC values of $8.9 \times 10^{3}-1.0 \times 10^{4} \mu \mathrm{mol}-\mathrm{Trolox}$ equivalents (TE)/g, SOSA of $1.5 \times 10^{4}-2.9 \times 10^{4}$ superoxide dismutase units/g, DPPH radical scavenging activity of $4.7 \times 10^{3}-5.5 \times 10^{3} \mu \mathrm{mol}-\mathrm{TE} / \mathrm{g}$, and reducing power of $6.5 \times 10^{2}-7.0 \times 10^{2}$ mg-ascorbic acid equivalents/g, and had excellent antioxidative properties.

\section{Keywords}

Eisenia Species, Ecklonia Species, Cultured E. kurome, H-ORAC, Phlorotannins, Radical Scavenging Activities

\footnotetext{
"Corresponding author.
} 


\section{Introduction}

Recently, many researchers are paying attention to the physiology of non-nutritive compounds included in botanical materials, especially the antioxidative properties and therapeutic effects of polyphenols. Polyphenols are a general term for an aromatic hydrocarbon which is combined with more than one hydroxyl group, and they are one of the most common secondary metabolites of plants. In the case of terrestrial plants, polyphenols are broadly divisible into two major groups: the condensed tannins [1] and the hydrolysable tannins [2]. Marine algal polyphenols, however, called phlorotannins, which have only been found to exist within brown algae, are formed by the polymerization of phloroglucinol (1,3,5-trihydroxybenzene) [3]-[6]. Phlorotannins can be divided into six categories: fucols, phlorethols, fucophlorethols, fuhalols, isofuhalols, and eckols, based on the type of structural linkages which result from the polymerization process, and on the number of hydroxyl groups [3] [5] [6]. From the brown algae distributed across the littoral regions of Japan, many kinds of phlorotannins, especially eckols, are chiefly isolated from Eisenia and Ecklonia species [4] [7]. Previously, we isolate phloroglucinol and eckols: eckol (phloroglucinol trimer), phlorofucofuroeckol A (a pentamer), dieckol and 8,8'-bieckol (hexamers) from the Eisenia and Ecklonia species, and report their inhibition of phospholipid peroxidation in the liposome system and scavenging activities of these phlorotannins against the superoxide anion and 1,1-diphenyl-2-picrylhydrazyl (DPPH) radicals [8]. In the concentration of $1 \mu \mathrm{M}$, the eckols show potent inhibition of phospholipid peroxidation in the liposome system that is more effective than the positive controls (epigallocatechin gallate, resveratrol, ascorbic acid, and $\alpha$-tocopherol) [8]. In addition, from the comparison of the $50 \%$ effective concentration value of tested samples, the scavenging activities of the eckols, including phlorofucofuroeckol A, dieckol and 8,8'-bieckol on DPPH and superoxide anion radicals are found to be around 2 - 10 times higher than those of catechin, ascorbic acid, and $\alpha$-tocopherol [8]. There is a report about superior antioxidative properties of phlorotannins in other studies. Ahn et al. [9] showed that phloroglucinol, eckol, and dieckol isolated from the brown alga Ecklonia cava had scavenging effects against alkyl and hydroxyl radicals. Kwon et al. [10] isolated bieckols (6,6'-bieckol, 6,8'-bieckol, and 8,8'-bieckol) from the brown alga Eisenia bicyclis and reported that their singlet oxygen quenching effects were superior to the effect of histidine, a wellknown singlet oxygen quencher. Thus, it can be concluded that the individual phlorotannin compounds from the Eisenia and Ecklonia species have prominent antioxidant properties as antioxidant polyphenols, as with the polyphenols of terrestrial plants.

Using the background of the scholarly research on phlorotannins, attempts at the utilization of phlorotannins are now occurring in the Japanese industrial fields of food, chemistry, cosmetology, and medicine. In order to promote the application of phlorotannins as natural antioxidants, the following two points become key: 1) more antioxidative property focused research analysis is needed of both phlorotannin extract (crude phlorotannins) and individual phlorotannin compounds, and 2) the supply of the brown algae, from which the phlorotannins are extracted, must be stabilized and secured. The main objective of this study is to evaluate the antioxidative properties of crude phlorotannins prepared from Japanese Eisenia species (Eisenia bicyclis and Eisenia arborea) and Ecklonia species (Ecklonia cava and Ecklonia kurome). The analysis of the antioxidative properties of the phlorotannins is accomplished by using several methods. Those methods include: the hydrophilic oxygen radical absorbance capacity (H-ORAC) method; the measurements of superoxide anion radical scavenging activity (SOSA) with electron spin resonance (ESR) method; and the 2,2-diphenyl-1-picrylhydrazyl (DPPH) radical scavenging activity method. Additionally, we determine the reducing power of the phlorotannins. Currently, $E$. kurome is cultivated as a supply source of phlorotannnins by applying the cultivation method of traditional edible seaweeds (e.g. Undaria pinnatifida and Saccharina japonica) in Kumamoto prefecture, Japan. However, no comprehensive study has yet been conducted in order to characterize and evaluate the antioxidative properties of phlorotannins obtained from cultured E. kurome. Therefore, the identification of major phlorotannin components in the natural plants of the Eisenia and Ecklonia species, and cultured plant of E. kurome, is carried out using liquid chromatography mass spectrometry (LC/MS) in this study. The availability of phlorotannins from the Japanese Eisenia and Ecklonia species as antioxidants is discussed.

\section{Materials and Methods}

\subsection{Algal Materials}

The brown algae E. bicyclis (Kjellman) Setchell, E. kurome Okamura, E. arborea Areschoug, and E. cava 
Kjellman, approximately 90 - $120 \mathrm{~cm}$ in length without any visible grazing or other tissue damage, were collected from along the coasts of the Itoshima peninsula $\left(33^{\circ} 37^{\prime} \mathrm{N}, 130^{\circ} 10^{\prime} \mathrm{E}\right)$ in Fukuoka prefecture, Itsuwa $\left(32^{\circ} 54^{\prime} \mathrm{N}, 130^{\circ} 13^{\prime} \mathrm{E}\right)$ in Kumamoto prefecture, and the Sakishima peninsula $\left(34^{\circ} 14^{\prime} \mathrm{N}, 136^{\circ} 50^{\prime} \mathrm{E}\right)$ in Mie prefecture, Japan, in 2013. The cultured E. kurome was purchased from the Fisheries Cooperative Association of Amakusa in Kumamoto prefecture, Japan, in 2013. The algae used for the extraction of phlorotannins were washed with filtered seawater, air-dried, and pulverized. The algal powders were stored at $-30^{\circ} \mathrm{C}$ until use.

\subsection{Chemicals}

2,2'-Azobis (2-amidinopropane) dihydrochloride (AAPH), DPPH, 6-hydroxy-2,5,7,8-tetramethylchroman-2carboxylic acid (Trolox), phloroglucinol, fluorescein, ultrapure water for LC/MS and methanol for LC/MS were all purchased from Wako Pure Chemical Industries (Osaka, Japan). 5,5-Dimethyl-1-pyrroline- $N$-oxide (DMPO) was purchased from LABOTEC (Tokyo, Japan). Diethylenetriaminepentaacetic acid (DETAPAC), hypoxanthine and superoxide dismutase (SOD) and were obtained from Sigma-Aldrich (MO, USA). Xanthine oxidase (XOD) was purchased from Roche Diagnostics (Basel, Switzerland). All reagents used in this study were of analytical grade.

\subsection{Extraction of Phlorotannins}

The extraction of the phlorotannins from the algal powder was carried out according to the method described in previous reports [8] [11] [12]. Briefly, the algal powder (25 g) was immersed in 80\% methanol (125 mL), and the mixture was shaken at $250 \mathrm{rpm}$ at room temperature for 3 hours with a shaking apparatus (IKA KS260 control, FR, Germany). After filtration with a qualitative filter (Grade 2, GE Healthcare Japan, Tokyo, Japan), 300 $\mathrm{mL}$ of $\mathrm{CHCl}_{3} /$ methanol (2:1, v/v) was added to the residue and shaken again at room temperature for 3 hours. These procedures were performed twice. The filtrate was mixed, and the mixture was partitioned between upper and lower layers by the addition of $\mathrm{CHCl}_{3}(200 \mathrm{~mL})$ and ultrapure water $(125 \mathrm{~mL})$. The upper layer, corresponding to the non-lipid fraction, was extracted twice with ethyl acetate $(300 \mathrm{~mL})$. The ethyl acetate fraction was evaporated in vacuo and the residue was made a crude phlorotannins (phlorotannin extract) [7] [8] [11]-[13].

\subsection{LC/MS Analyses}

The separation and identification of phlorotannins was carried out using an LC-MS system with an Inertsil ODS-3 column (250 mm $\times 4.6$ mm I.D., $4 \mu \mathrm{m}$, GL Science, Tokyo, Japan). The LC-MS system consisted of a 6120 quadrupole mass spectrometer (Agilent Technologies, CA, USA), a 1260 Infinity LC (Agilent Technologies, CA, USA), and an OpenLAB Chromatography Data System ChemStation (Agilent Technologies, CA, USA). Elution was performed at a flow rate of $1.0 \mathrm{~mL} / \mathrm{min}$ using a linear gradient from $20 \%$ methanol to $100 \%$ methanol for 20 minutes, and maintained for 20 minutes [7] [8] [13]-[15]. The UV detector was set at $290 \mathrm{~nm}$. Each phlorotannin was monitored by electrospray ionization mass spectrometry (ESI-MS) analysis in negative mode. Other experimental conditions for the mass spectrometer were as follows: dry gas, $12.0 \mathrm{~L} / \mathrm{min}$; nebulizer, $35 \mathrm{psi}$; dry temperature, $250^{\circ} \mathrm{C}$; vaporizer, $200^{\circ} \mathrm{C}$; scan from $\mathrm{m} / \mathrm{z} 100$ to $\mathrm{m} / \mathrm{z} 1300$. The peaks of phloroglucinol (m/z 126), eckol (m/z 372), fucofuroeckol A ( $\mathrm{m} / \mathrm{z} 478)$, phlorofucofuroeckol A ( $\mathrm{m} / \mathrm{z} 602)$, dieckol $(\mathrm{m} / \mathrm{z} 742)$ and 8,8'-bieckol ( $\mathrm{m} / \mathrm{z}$ 742) were detected using a mass spectrometer with a selected ion monitoring (SIM) detector.

\subsection{H-ORAC Assay}

The H-ORAC assay of crude phlorotannins was performed according to the method of Wu et al. [16] with a slight modification. Each of the crude phlorotannins was directly dissolved in 50\% ethanol and diluted with a 75 $\mathrm{mM}$ phosphate buffer ( $\mathrm{pH}$ 7.0). Trolox standards, fluorescein, and AAPH solutions were prepared by dissolving them in the phosphate buffer. The measurement was carried out using a 96-well black plate (Costar 3915, Corning, NY, USA) with plate seal (Q-Stick adhesive sheets for qPCR, 4 titude, Surrey, UK), and a 96-well microplate reader (Infinite 200, Tecan Japan, Kanagawa, Japan). The fluorescence intensity was monitored every 2 minutes for 90 minutes at excitation and emission wavelengths of 485 and $528 \mathrm{~nm}$ respectively on the microplate reader. The area under the curve (AUC) of the fluorescence decay after the addition of AAPH solution was calculated for each well. The net AUC was calculated by subtracting the AUC for the phlorotannins, or Trolox standard, from that of the blank. A calibration curve was constructed from the net AUCs of the Trolox standard 
solutions. The H-ORAC value for each sample was calculated on the basis of the standard curve for Trolox, and expressed as $\mu \mathrm{mol}$ of Trolox equivalents (TE) per gram (dry weight) of the phlorotannins. The final ORAC values were expressed as the mean of three independent measurements.

\subsection{Determination of SOSA}

Superoxide anion was generated by hypoxanthine-XOD system. Each of the crude phlorotannins, DETAPAC, XOD and SOD were dissolved separately in $0.1 \mathrm{M}$ phosphate buffer $(\mathrm{pH} 7.8)$. DMPO $(15 \mu \mathrm{L}), 5.5 \mathrm{mM}$ DETAPAC (35 $\mu \mathrm{L}), 2 \mathrm{mM}$ hypoxanthine $(50 \mu \mathrm{L})$ and the sample solution $(50 \mu \mathrm{L})$ were mixed in the test tube. Fifty $\mu \mathrm{L}$ of XOD $(0.4 \mathrm{U} / \mathrm{mL})$ was added to the mixture, and this was then transferred to an ESR flat cell. The recording of ESR spectrum was started 1 minute after XOD was added to the mixture. The measurement conditions of ESR (Multi-Analyzer MA10, JEOL, Tokyo, Japan) were as follows: magnetic field $335.0 \pm 5 \mathrm{mT}$; microwave power $8.0 \mathrm{~mW}$; modulation frequency $100 \mathrm{kHz}$; modulation amplitude $0.8 \times 0.1 \mathrm{mT}$; sweep time 2 minutes; response time 0.1 second. Intensity of the DMPO-OOH signal was measured relative to that of manganese oxide as an internal standard. The data was expressed as units of SOD equivalents per gram (dry weight) of the phlorotannins.

\subsection{Determination of DPPH Radical Scavenging Activity}

The DPPH working solution was mixed with $0.4 \mathrm{mM}$ DPPH in ethanol/400 mM MES buffer (pH 6.1)/ultrapure water (4:1:3, v/v). Ten $\mu \mathrm{L}$ of the sample solution was added to the $190 \mu \mathrm{L}$ of the DPPH working solution. After incubation for 20 minutes at room temperature in a light-proof enclosure, the absorbance was measured at 517 nm using a U-1900 spectrophotometer (Hitachi High Technologies, Tokyo, Japan). The relative activity of the phlorotannins was calculated in relation to the activity of Trolox as a positive control. The data was expressed as $\mu \mathrm{mol}$ of TE per gram (dry weight) of the phlorotannins.

\subsection{Determination of Reducing Power}

Total reducing power of phlorotannins was measured as described by Zhu et al. [17] with a slight modification. Each of the crude phlorotannins was dissolved in methanol. Fifty $\mu \mathrm{L}$ of the sample solution $(0.1 \mathrm{mg} / \mathrm{mL})$ was mixed with $0.2 \mathrm{M}$ phosphate buffer $(\mathrm{pH} 6.6,250 \mu \mathrm{L})$ and $1 \%$ potassium ferricyanide $(250 \mu \mathrm{L})$. The reaction mixture was incubated at $50^{\circ} \mathrm{C}$ for 30 minutes and $250 \mu \mathrm{L}$ of $10 \%$ trichloroacetic acid was then added. The mixture was centrifuged at $4500 \mathrm{~g}$ for 10 minutes. The supernatant $(200 \mu \mathrm{L})$ was mixed with $0.1 \%$ ferric chloride $(40 \mu \mathrm{L})$ and the absorbance was measured at $700 \mathrm{~nm}$. The relative activity of the phlorotannins was calculated in relation to the activity of L-ascorbic acid used as a positive control. The data were expressed as milligrams of ascorbic acid equivalents (ASE) per gram (dry weight) of the phlorotannins.

\section{Results and Discussion}

\subsection{LC/MS Analysis of Phlorotannins}

The yield of crude phlorotannins among E. bicyclis, E. arborea, E. cava, E. kurome from Fukuoka, E. kurome from Kumamoto, and cultured E. kurome were 6.1, 2.4, 2.4, 7.9, 4.0 and 3.7\% of the algal powder of each, respectively. All of the crude phlorotannins were used for the following LC/MS analysis and antioxidant experiments.

The differences and similarities in crude phlorotannins from natural plants of Eisenia species (E. bicyclis and E. arborea), Ecklonia species (E. cava, and E. kurome), and cultured E. kurome were analyzed by LC/MS. Figure 1 shows a UV chromatogram of phlorotannins from the tested brown algae, recorded at $290 \mathrm{~nm}$. Figure 2 presents a total ion chromatogram (TIC) of phlorotannins in negative mode of ESI-MS analysis. As shown in Figure 1, a distinct separation of each of the components in the crude phlorotannins was obtained by using a linear gradient system ranging from $20 \%$ methanol to $100 \%$ methanol within 20 minutes. The known eckols [4] [7] [18] are shown in Figure 3. Those eckols are: eckol (peak b); fucofuroeckol A (peak e); phlorofucofuroeckol A (peak d); dieckol (peak c); and 8,8'-bieckol (peak a). They were identified using a mass spectrometer with SIM detector. It is possible that the mass spectrometer has a high sensitivity for detecting only the specified mass range in SIM mode. The known eckols were detected in all the crude phlorotannins from the tested brown 


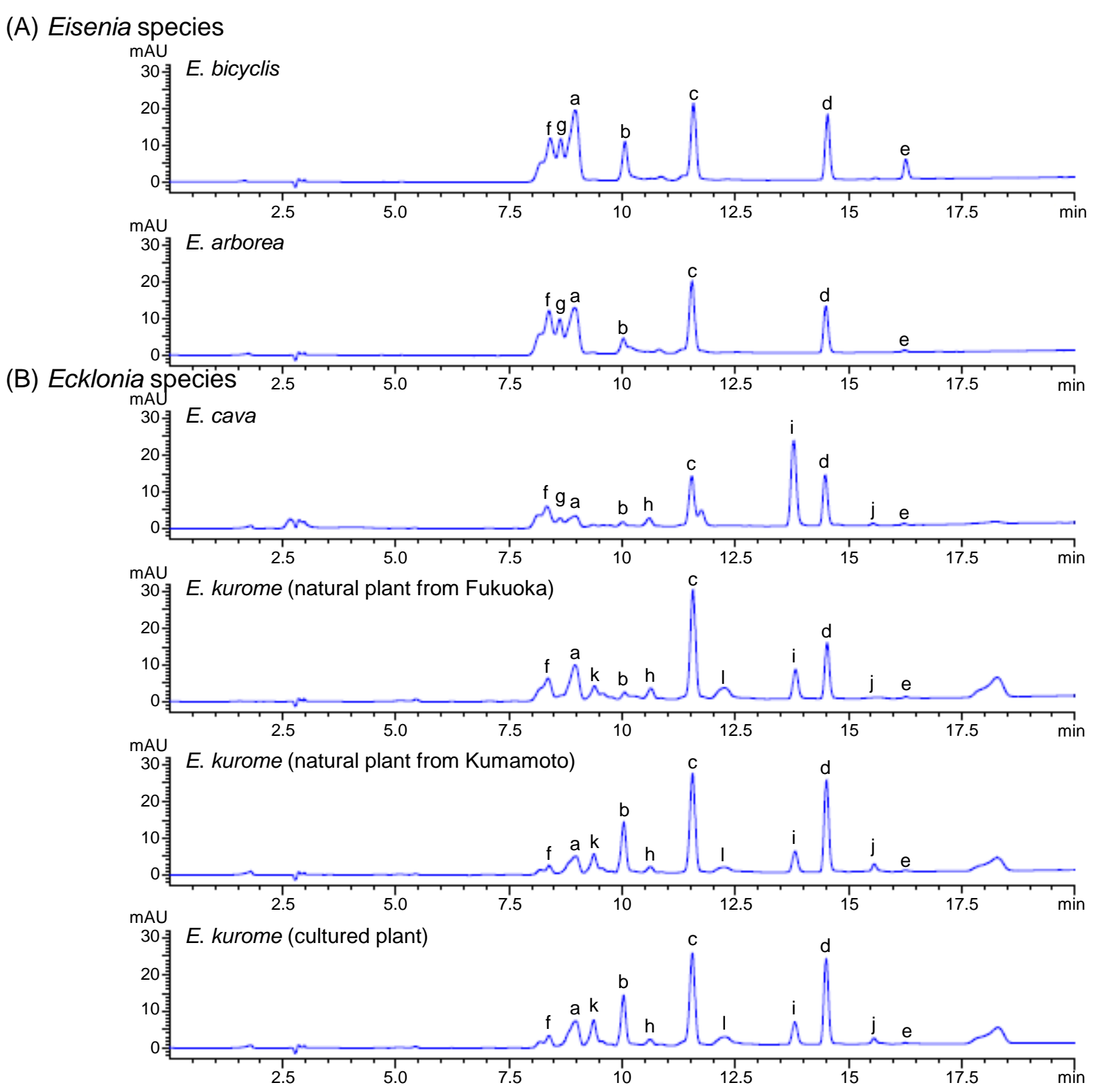

Figure 1. HPLC of crude phlorotannins from Japanese Eisenia and Ecklonia species. (A) Eisenia species, (B) Ecklonia species. Ten $\mu \mathrm{L}$ of each crude phlorotannins solution $(0.5 \mathrm{mg} / \mathrm{mL})$ was injected into the LC-MS system. Conditions for the LC/MS are described in the materials and methods. a, 8,8'-bieckol; b, eckol; c, dieckol; d, phlorofucofuroeckol A; e, fucofuroeckol A; f, phloroglucinol heptamer; g and h, phloroglucinol hexamers; i and l, phloroglucinol octamers; j, phloroglucinol trimer; $\mathrm{k}$, phloroglucinol tetramer.

algae as common components (Figure 1 and Figure 2). Among the eckols, eckol (peak b) and dieckol (peak c) were detected as the prominent peaks in the TIC of tested samples (Figure 2). It was clarified that fucofuroeckol A (peak e) was a notable compound in the crude phlorotannins of E. bicyclis (Figure 1 and Figure 2).

It was indicated that the obtained chromatograms of phlorotannins had some differences among the Eisenia and Ecklonia species. In the case of the Ecklonia species, the crude phlorotannins of E. kurome contained unidentified peaks i and l between dieckol (peak c) and phlorofucofuroeckol A (peak d). In addition, the peak i was commonly detected as the characteristic component of crude phlorotannins in the Ecklonia species (Figure 1), and these are markedly different from Eisenia species (Figure 1). In the negative ion ESI-MS spectrum of peaks i and l, the $[\mathrm{M}-\mathrm{H}]^{-}$ion peak at $\mathrm{m} / \mathrm{z} 973$ (relative intensity 100\%) was observed. It is hypothesized that each of these compounds is a phloroglucinol octamer which corresponds to the molecular formula $\mathrm{C}_{48} \mathrm{H}_{30} \mathrm{O}_{23}$. Peak k was 
(A) Eisenia species
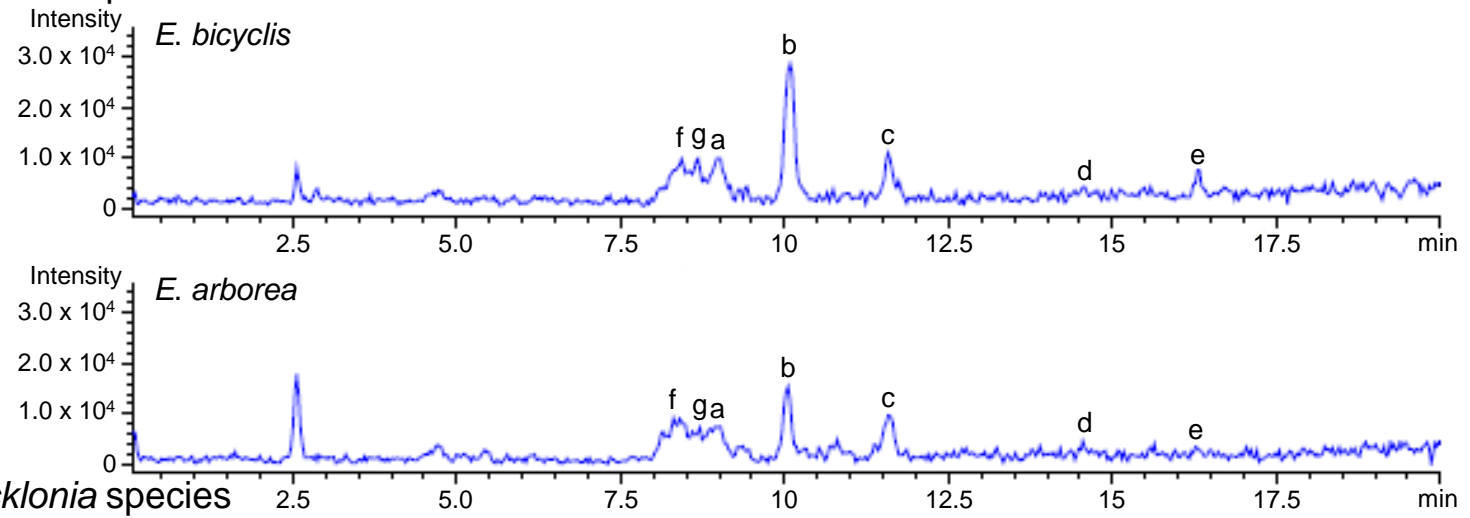

(B) Ecklonia species 25
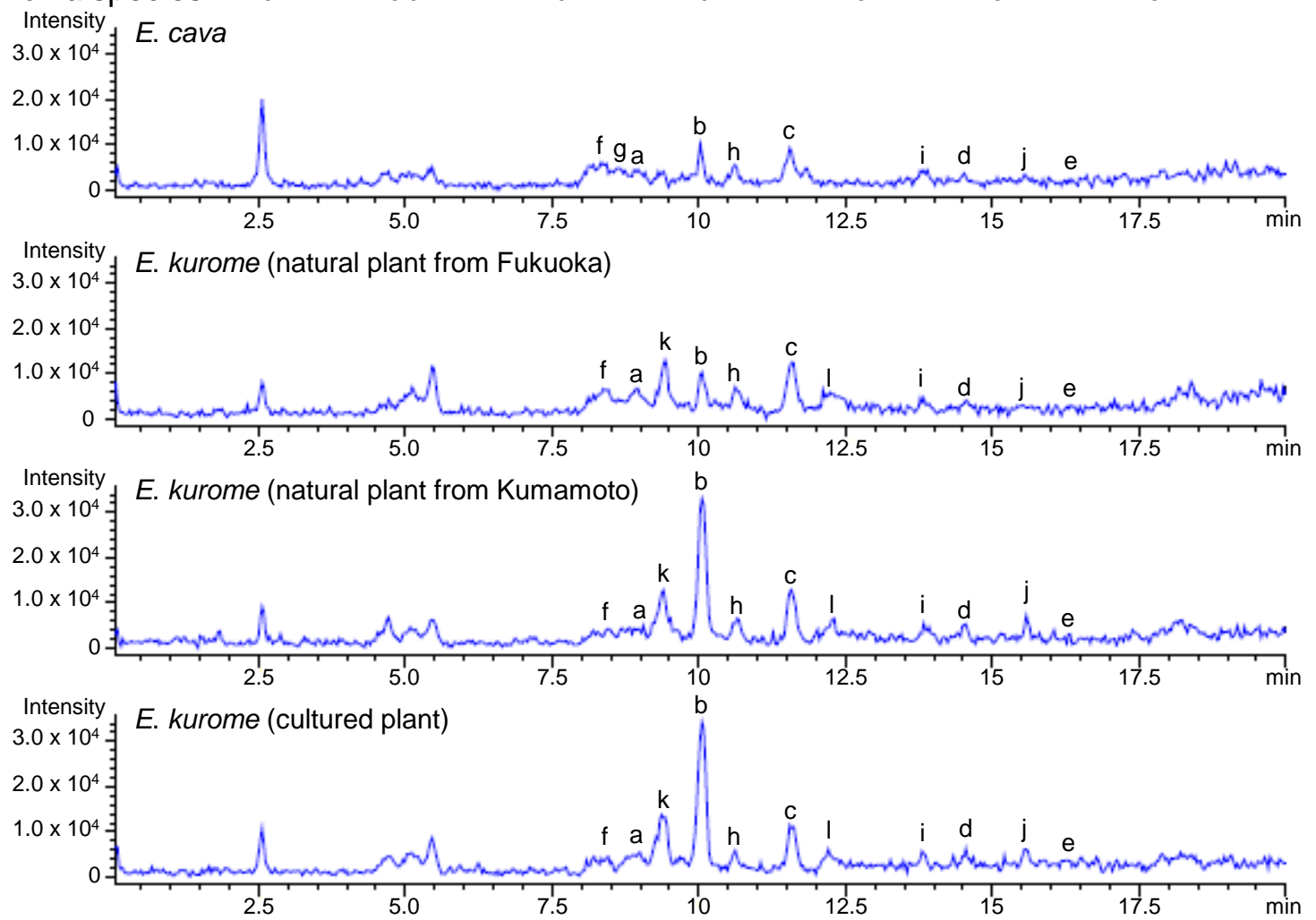

Figure 2. TIC of crude phlorotannins from Japanese Eisenia and Ecklonia species. (A) Eisenia species, (B) Ecklonia species. a, 8,8'-bieckol; b, eckol; c, dieckol; d, phlorofucofuroeckol A; e, fucofuroeckol A; f, phloroglucinol heptamer; g and h, phloroglucinol hexamers; i and l, phloroglucinol octamers; j, phloroglucinol trimer; k, phloroglucinol tetramer.

detected only in E. kurome and it showed an $[\mathrm{M}-\mathrm{H}]^{-}$ion peak at $\mathrm{m} / \mathrm{z} 495$ (relative intensity 100\%). It is hypothesized that the compound is a phloroglucinol tetramer. In addition, the other minor compounds detected only in chromatograms of Ecklonia species were as follows: peak h with $\mathrm{m} / \mathrm{z} 743$ (relative intensity 100\%) and peak j with $\mathrm{m} / \mathrm{z} 369$ (relative intensity 100\%).

Of the other two major peaks ( $\mathrm{f}$ and g), peak $\mathrm{f}$ was detected in all chromatograms of the Eisenia and Ecklonia species (Figure 1 and Figure 2). Peak $\mathrm{f}$ showed an $[\mathrm{M}-\mathrm{H}]^{-}$ion peak at $\mathrm{m} / \mathrm{z} 865$ (relative intensity 100\%) and characteristic ion at $m / z 741$ (M-125) and $m / z 495$ (M-371) in the negative ESI-MS spectrum. Judging from the result, it is hypothesized that peak $\mathrm{f}$ is a phloroglucinol heptamer with a molecular weight of 866. As a result of analysis, peak g, which was detected in the Eisenia species and E. cava, had an $[\mathrm{M}-\mathrm{H}]^{-}$ion peak at $\mathrm{m} / \mathrm{z} 741$ (relative intensity 100\%) and characteristic ion at $\mathrm{m} / \mathrm{z} 495$ (M-247) in common with other known eckols [4]. It is hypothesized that the compound is a phloroglucinol hexamer which corresponds to the molecular formula $\mathrm{C}_{36} \mathrm{H}_{22} \mathrm{O}_{18}$. 


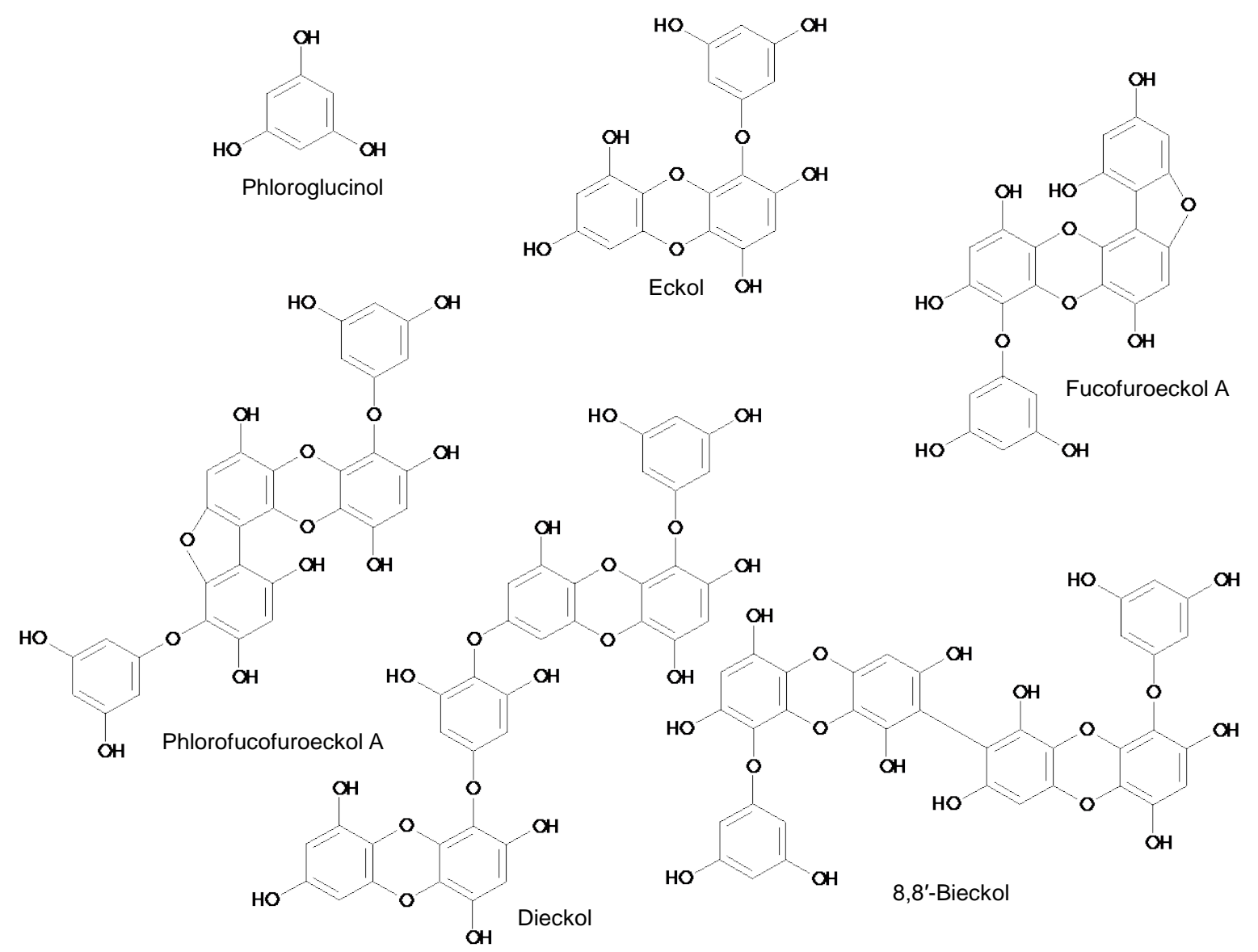

Figure 3. Chemical structures of phloroglucinol and eckols from Japanese Eisenia and Ecklonia species.

From the evidence obtained in the present study, it is suggested that the phlorotannins of the Japanese Eisenia and Ecklonia species are composed of compounds being observed between 3 - 7 phloroglucinol units and 3 - 8 phloroglucinol units, respectively. Kellogg et al. [19] reported that phlorotannins from the brown alga Fucus distichus were categorized as fucophlorethol isomers ranging from 3 to 18 phloroglucinol units. It has become apparent that phlorotannins of $F$. vesiculosus and $F$. serratus consist of phloroglucinol units from 3 to 13 and phloroglucinol units from 3 to 16, respectively [20]. Compared with the phlorotannins of Fucaceae, it was found that the phlorotannins of the Eisenia and Ecklonia species were composed of compounds with a low degree of polymerization of phloroglucinol. In other studies on phlorotannins, it is reported that Eisenia and Ecklonia species produce some phloroglucinol hexamers such as 8,4'”'-dieckol, 6,6'-bieckol, and 7,7'-bieckol [3] [10]. Kang et al. [21] [22] identified two phlorotannins; pyrogallol-phloroglucinol-6,6'-bieckol (molecular weight 974) and 2,7' '-phloroglucinol-6,6'-bieckol (molecular weight 974) from the diethyl ether fraction of the E. cava. Recently, Yotsu-Yamashita et al. [23] isolated two novel phlorotannins with a molecular weight of 974, 974-A and 974-B, from the ethyl acetate fraction of the E. kurome and showed their antioxidant activities. Because the mass number of each compound and the algal species are completely corresponding, the major unidentified phloroglucinol oligomers detected by this study (phloroglucinol hexamer in Eisenia species and E. cava and phloroglucinol octamers in Ecklonia species), might be contained in the group of compounds reported in the literature of Kang et al. [21] [22] and Yotsu-Yamashita et al. [23]. It is well-known that the plants of Eisenia and Ecklonia species are perennial sporophytes. After germination, the E. kurome, which is cultivated in Kumamoto, is harvested at about ten months. Therefore, the cultured $E$. kurome used in this study, can be considered to be a young plant of E. kurome. As shown in Figure 1 and Figure 2, there was no difference in the composition of the phlorotannins among the examined E. kurome, regardless of habitat or stage of growth. Therefore, as with the natural plants of Eisenia and Ecklonia species, it was clarified that the cultured E. kurome can be used as a supply source of phlorotannins. 


\subsection{H-ORAC Assay of Phlorotannins}

Recently, the oxygen radical absorbance capacity (ORAC) assay is widely used as one of the quantitative standards that show the antioxidative potencies of antioxidants (e.g. vitamins and polyphenols), vegetables, fruits, and their processed products and beverages (e.g. tea and wine) [16] [24]. In the case of phlorotannins or phlorotannin-rich algae, Parys et al. [25] isolated trifucodiphlorethol A, trifucotriphlorethol A, and fucotriphlorethol A from the brown alga Fucus vesiculosus and reported their H-ORAC values. Apostolidis et al. [26] prepared hot water extract from the brown alga Ascophyllum nodosum which is rich in phlorotannins, and determined it's H-ORAC value. Thus, reports on the H-ORAC assay of phlorotannins or phlorotannin-rich algae have focused on Fucales [25]-[29] such as F. vesiculosus and A. nodosum, and the H-ORAC values of phlorotannins from Eisenia and Ecklonia species are still obscure. Table 1 shows the H-ORAC value of each of the crude phlorotannins isolated from the Japanese Eisenia and Ecklonia species. Among the tested samples, the crude phlorotannins of $E$. bicyclis displayed the most superior antioxidant activity with H-ORAC values of $1.0 \times 10^{4} \mu \mathrm{mol}-\mathrm{TE} / \mathrm{g}$. In contrast, those of E. arborea, E. cava and E. kurome had almost the same values at $9.7 \times 10^{3}, 9.7 \times 10^{3}$, and $9.6 \times 10^{3} \mu \mathrm{mol}-\mathrm{TE} / \mathrm{g}$, respectively. The H-ORAC value of cultured $E$. kurome was slightly lower than those of natural plants of E. kurome (Table 1). Wang et al. [27] determined the ORAC value of 10 kinds of Icelandic seaweeds. In the literature [27], it was reported that the ORAC values of $70 \%$ acetone extracts from $F$. vesiculosus, F. serratus and A. nodosum were 2567, 2545 and $1417 \mu$ mol-TE/g, respectively. In the comparison of simple numerical values, the ORAC value of the crude phlorotannins form Eisenia and Ecklonia species exceed those of Fucaceae. Thus, it was suggested that the crude phlorotannins of the Eisenia and Ecklonia species, including cultured $E$. kurome, had excellent peroxyl radical scavenging activity.

In the preceding report [18], we purified five eckols shown in Figure 3 from crude phlorotannins of E. bicyclis, and calculated their H-ORAC values. Among the samples, it was found that the compounds contained more than phloroglucinol tetramers. The compounds contained notable levels of: fucofuroeckol A (molecular weight 478); phlorofucofuroeckol A (molecular weight 602); dieckol (molecular weight 742); and 8,8'-bieckol (molecular weight 742). These additional phlorotannins had superior antioxidant activity with H-ORAC values in the range of 8.62 - $10.22 \mathrm{~mol}-\mathrm{TE} / \mathrm{mol}$. Although eckol (phloroglucinol trimer, molecular weight 372) had little antioxidant activity (H-ORAC value: $4.97 \mathrm{~mol}-\mathrm{TE} / \mathrm{mol}$ ) in the tested compounds, it was more effective than L-ascorbic acid and epigallocatechin gallate (H-ORAC values: 0.76 and $4.65 \mathrm{~mol}-\mathrm{TE} / \mathrm{mol}$ ). Ishimoto et al. [30] reported the H-ORAC values of 43 terrestrial polyphenols and their metabolites. Compared with their H-ORAC values in the report, the known eckols, in particular fucofuroeckol A and dieckol, had H-ORAC values (9.82 and $10.22 \mathrm{~mol}-\mathrm{TE} / \mathrm{mol}$ ) that were higher than antioxidant polyphenols (ellagitannins, condensed tannins, chlorogenic acids, catechins, and flavonoids). Therefore, it was suggested that the polymeric eckols, which were included in Eisenia and Ecklonia species, had more potent peroxyl radical scavenging activity than many representative polyphenols (e.g. epigallocatechin gallate, kaempferol, quercetin, myricetin, chlorogenic acid, caffeic acid, gallic acid, and ellagic acid).

\begin{tabular}{|c|c|}
\hline \multirow{2}{*}{ Brown algae } & H-ORAC values ${ }^{a}$ \\
\hline & $(\mu \mathrm{mol}-\mathrm{TE} / \mathrm{g})$ \\
\hline \multicolumn{2}{|l|}{ Eisenia species } \\
\hline E. bicyclis & $1.0 \times 10^{4}$ \\
\hline E. arborea & $9.7 \times 10^{3}$ \\
\hline \multicolumn{2}{|l|}{ Ecklonia species } \\
\hline E. cava & $9.7 \times 10^{3}$ \\
\hline E. kurome (natural plant from Fukuoka) & $9.6 \times 10^{3}$ \\
\hline E. kurome (natural plant from Kumamoto) & $9.6 \times 10^{3}$ \\
\hline E. kurome (cultured plant) & $8.9 \times 10^{3}$ \\
\hline
\end{tabular}

${ }^{\mathrm{a}}$ All the data are expressed as the mean from three independent measurements. 


\subsection{SOSA, DPPH Radical Scavenging Activity and Reducing Power of Phlorotannins}

In addition, to evaluate antioxidative properties of the crude phlorotannins, we measure SOSA using ESR method and DPPH radical scavenging activity. As for the superoxide anion radical, when thinking about the mechanism of formation of reactive oxygen species in a living cell, it is thought to play the role of the precursor of other reactive oxygen species, though its reactivity is low [31]. Therefore, it is thought that the generation of excessive superoxide anion radical causes the cell damage because it increases other free radicals and oxidizing agents [31]. SOD, which exists in the cytoplasm, is effectively able to disproportionate the superoxide anion radical into dioxygen, which has the role in decreasing oxidant stress [31]. Because most of the antioxidants have radical scavenging activity, it is considered that measurement of SOSA (i.e. SOD-like activity) is an important index that evaluates the antioxidative property of the target sample. Table 2 shows the SOSA of the crude phlorotannins from the Eisenia and Ecklonia species measured by ESR spectroscopy. Among the tested samples, the crude phlorotannins of E. arborea show the highest SOSA with a value of $2.9 \times 10^{4}$ SOD units/g (Table 2). As for the crude phlorotannins from E. bicylis and three kinds of $E$. kurome, including cultured $E$. kurome, each value of SOSA is slightly lower than that of $E$. arborea and the ranges are from $2.3 \times 10^{4}$ to $2.6 \times$ $10^{4} \mathrm{SOD}$ units/g (Table 2). E. cava is the lowest in the examined sample group and the value of $1.5 \times 10^{4} \mathrm{SOD}$ units/g is obtained through the SOSA of crude phlorotannins. Because the data on SOSA of crude phlorotannins or brown algal extracts that use the ESR method are insufficient, the results in this study are not able to be compared with the value of SOSA of other phlorotannin-rich algae. However, from the comparison with the value of SOSA reported in the literature for terrestrial plants [32], vegetables [33], and Chinese herbal medicine [34], it has been found that the SOSA of crude phlorotannins from the Eisenia and Ecklonia species exceeded many of them.

The DPPH radical is an artificial and stable radical, and a material that does not exist in vivo [35]. Because a peculiar color of the DPPH radical discolors if an unpaired electron is trapped, the DPPH radical scavenging ability can be determined by measuring the level of discoloration at the wavelength of $517 \mathrm{~nm}$ [35]. For these reasons, the DPPH radical has been widely used to test the ability of compounds to act as free radical scavengers and to evaluate the antioxidant activity of foods and plant extracts. The data of the DPPH radical scavenging activities of the tested samples are shown in Table 2. The radical scavenging activities of crude phlorotannins of $E$. bicyclis and cultured E. kurome are the highest in the tested samples, and both results are $5.5 \times 10^{3} \mu \mathrm{mol}-\mathrm{TE} / \mathrm{g}$ (Table 2). In the crude phlorotanins of other Ecklonia species, the DPPH radical scavenging activity was shown in decreasing order in the natural plant of E. kurome from Kumamoto $\left(5.3 \times 10^{3} \mu \mathrm{mol}-\mathrm{TE} / \mathrm{g}\right)$, natural plant of $E$. kurome from Fukuoka $\left(5.0 \times 10^{3} \mu \mathrm{mol}-\mathrm{TE} / \mathrm{g}\right)$, and E. cava $\left(4.7 \times 10^{3} \mu \mathrm{mol}-\mathrm{TE} / \mathrm{g}\right)($ Table 2$)$. This order is similar to the result of SOSA.

The reducing power assay provides a measure of the ability of the target sample to donate electrons and to

Table 2. Radical scavenging activities and reducing power of crude phlorotannins from Japanese Eisenia and Ecklonia species.

\begin{tabular}{lccc}
\hline \multicolumn{1}{c}{ Brown algae } & SOSA & $\begin{array}{c}\text { DPPH radical } \\
\text { scavenging activity }\end{array}$ & \begin{tabular}{c} 
Reducing power \\
\cline { 2 - 4 }
\end{tabular} \\
\cline { 2 - 4 } & $(\text { SOD unit/g) })^{\mathrm{a}}$ & $(\mu \mathrm{mol}-\mathrm{TE} / \mathrm{g})^{\mathrm{a}}$ & $(\mathrm{mg}-\mathrm{ASE} / \mathrm{g})^{\mathrm{a}}$ \\
\hline $\begin{array}{l}\text { Eisenia species } \\
\text { E. bicyclis }\end{array}$ & $2.6 \times 10^{4}$ & $5.5 \times 10^{3}$ & $6.6 \times 10^{2}$ \\
E. arborea & $2.9 \times 10^{4}$ & $5.0 \times 10^{3}$ & $6.5 \times 10^{2}$ \\
Ecklonia species & & & \\
E. cava & $1.5 \times 10^{4}$ & $4.7 \times 10^{3}$ & $6.5 \times 10^{2}$ \\
E. kurome (natural plant from Fukuoka) & $2.3 \times 10^{4}$ & $5.0 \times 10^{3}$ & $6.5 \times 10^{2}$ \\
E. kurome (natural plant from Kumamoto) & $2.4 \times 10^{4}$ & $5.3 \times 10^{3}$ & $6.9 \times 10^{2}$ \\
E. kurome (cultured plant) & $2.6 \times 10^{4}$ & $5.5 \times 10^{3}$ & $7.0 \times 10^{2}$ \\
\hline
\end{tabular}

${ }^{\mathrm{a}}$ All the data are expressed as the mean from three independent measurements. 
reduce the oxidized intermediates of the peroxidation process [17] [29]. In this study, the reducing power of crude phlorotannins from the Eisenia and Ecklonia species to reduce ferric ions was determined using L-ascor bic acid as a reference compound. Among the tested sample, the highest reducing power was observed in the crude phlorotannins of cultured E. kurome and the natural plant of E. kurome from Kumamoto, and the data were $7.0 \times 10^{2}$ and $6.9 \times 10^{2} \mathrm{mg}$-ASE/g, respectively (Table 2). In the case of other brown algae (E. bicylis, $E$. arborea, E. cava and E. kurome from Fukuoka), each of the crude phlorotannins display almost the same data with the values in the range of $6.5 \times 10^{2}-6.6 \times 10^{2} \mathrm{mg}$-ASE/g. In other marine algal species, there are reports about the reducing power of ethyl acetate fraction from red algae Polysiphonia urceolata and Rhodomela confervoides and the data were 383 and 426 mg-ASE/g [36] [37]. In the case of phlorotannin-rich algae, Wang et al. [38] reported that the ethyl acetate fraction (i.e. crude phlorotannins) of $F$. vesiculosus extract was fractionated by gel filtration and ultrafiltration and each fraction possessed the high DPPH radical scavenging activity and reducing power. The data of reducing power, which was reported in the literature, were in the range of 717.8 $910.7 \mathrm{mg}-\mathrm{ASE} / \mathrm{g}$ [38]. As pointed out the section of LC/MS analysis, it has become apparent that phlorotannins of the F. vesiculosus consists of phloroglucinol units from 3 to 13 [19]. The phlorotannins of the $F$. vesiculosus contains more of the compounds of the oligomer and polymer than the phlorotannins of Japanese Eisenia and Ecklonia species. The difference of the reducing power might originate in the difference between molecular weight and the amount of the contained phloroglucinol oligomers and polymers. As for the antioxidative property, it is pointed out that the result might be different depending on the differences of the analytical method, and radicals or reactive oxygen species that become target of the sample. However, in the case of phlorotannins from Eisenia and Ecklonia species, all the analytical data of H-ORAC assay, SOSA, DPPH-radical scavenging activity, and the reducing power, showed a high level of antioxidative property.

In conclusion, the following two points in this study are understood: 1) the antioxidative properties and the reducing power of crude phlorotannins from Japanese Eisenia and Ecklonia species are of superior levels compared with other algal extracts and botanical extracts, and 2) as for the cultured E. kurome, the composition of contained phlorotannins and it's antioxidative properties are also the same as the natural plant of E. kurome. At present, the correlation of the structure and the antioxidative properties of the phlorotannins has not been made clear. From the results of our previous studies, it is suggested that the number of phenolic hydroxyl groups attached to the eckol skeleton play an important role in the antioxidant activities. It is demonstrated that the crude phlorotannins of the Eisenia and Ecklonia species are composed of polymeric eckols from the LC/MS analysis in this study. It seems that this data indirectly proves excellent antioxidative properties in vitro of the crude phlorotannins. Recently, there are reports concerning the administration and the influence of phlorotannins on the living body. Nagayama et al. [12] demonstrated that phlorotannins isolated from E. kurome had no toxicity following oral administration to mice. Shin et al. [39] reported that phlorotannins extracted from E. cava contributed to a lowering of body fat and serum lipid parameters, such as total and low density lipoprotein cholesterols, in human study. The evidence obtained in each of the previously cited studies suggests that phlorotannins are prominent and safe natural antioxidants, and that the Japanese Eisenia and Ecklonia species, including cultured E. kurome, are useful as a novel functional food, or as a plant material with antioxidative properties.

\section{Acknowledgements}

This work is supported by the Japan Science and Technology Agency, CREST. We thank Dr. Shigeo Kawaguchi of the Graduate School of Kyushu University for identifying the Eisenia and Ecklonia species. We also acknowledge that the English is revised by Mr. Andrew Rother and Mr. Shane LeGros (Eigo Step, Tsu, Japan).

\section{References}

[1] Schofield, P., Mbugua, D.M. and Pell, A.N. (2001) Analysis of Condensed Tannins: A Review. Animal Feed Science and Technology, 91, 21-40. http://dx.doi.org/10.1016/S0377-8401(01)00228-0

[2] Mueller-Harvey, I. (2001) Analysis of Hydrolysable Tannins. Animal Feed Science and Technology, 91, 3-20. http://dx.doi.org/10.1016/S0377-8401(01)00227-9

[3] Ragan, M.A. and Glombitza, K.W. (1986) Phlorotannins, Brown Algal Polyphenols. Progress in Phycological Research, 4, 129-241.

[4] Nakamura, T., Nagayama, K., Uchida, K. and Tanaka, R. (1996) Antioxidant Activity of Phlorotannins Isolated from the Brown Alga Eisenia bicyclis. Fisheries Science, 62, 923-926. 
[5] Amsler, C.D. and Fairhead, V.A. (2005) Defensive and Sensory Chemical Ecology of Brown Algae. Advances in Botanical Research, 43, 1-91. http://dx.doi.org/10.1016/S0065-2296(05)43001-3

[6] Martínez, J.H.I. and Castañeda, H.G.T. (2013) Preparation and Chromatographic Analysis of Phlorotannins. Journal of Chromatographic Science, 51, 825-838. http://dx.doi.org/10.1093/chromsci/bmt045

[7] Shibata, T., Kawaguchi, S., Hama, Y., Inagaki, M., Yamaguchi, K. and Nakamura, T. (2004) Local and Chemical Distribution of Phlorotannins in Brown Algae. Journal of Applied Phycology, 16, 291-296. http://dx.doi.org/10.1023/B:JAPH.0000047781.24993.0a

[8] Shibata, T., Ishimaru, K., Kawaguchi, S., Yoshikawa, H. and Hama, Y. (2008) Antioxidant Activities of Phlorotannins Isolated from Japanese Laminariaceae. Journal of Applied Phycology, 20, 705-711. http://dx.doi.org/10.1007/s10811-007-9254-8

[9] Ahn, G.N., Kim, K.N., Cha, S.H., Song, C.B., Lee, J., Heo, M.S., Yeo, I.K., Lee, N.H., Jee, Y.H., Kim, J.S., Heu, M.S. and Jeon, Y.J. (2007) Antioxidant Activities of Phlorotannins Purified from Ecklonia cava on Free Radical Scavenging Using ESR and $\mathrm{H}_{2} \mathrm{O}_{2}$-Mediated DNA Damage. European Food Research and Technology, 226, 71-79. http://dx.doi.org/10.1007/s00217-006-0510-y

[10] Kwon, T.H., Suh, H.J., Lee, I.K., Yun, B.S., Kim, T.W., Hwang, D.I., Kim, Y.J., Kim, M.J., Kwon, O.O., Kim, C.G. and Park, N.H. (2013) Determination of Singlet Oxygen Quenching and Antioxidant Activity of Bieckols Isolated from the Brown Alga Eisenia bicyclis. European Food Research and Technology, 237, 501-508. http://dx.doi.org/10.1007/s00217-013-2017-7

[11] Nagayama, K., Shibata, T., Fujimoto, K., Honjo, T. and Nakamura, T. (2003) Algicidal Effect of Phlorotannins from the Brown Alga Ecklonia kurome on Red Tide Microalgae. Aquaculture, 218, 601-611. http://dx.doi.org/10.1016/S0044-8486(02)00255-7

[12] Nagayama, K., Iwamura, Y., Shibata, T., Hirayama, I. and Nakamura, T. (2002) Bactericidal Activity of Phlorotannins from the Brown Alga Ecklonia kurome. Journal of Antimicrobial Chemotherapy, 50, 889-893. http://dx.doi.org/10.1093/jac/dkf222

[13] Shibata, T., Hama, Y., Miyasaki, T., Ito, M. and Nakamura, T. (2006) Extracellular Secretion of Phenolic Substances from Living Brown Algae. Journal of Applied Phycology, 18, 787-794. http://dx.doi.org/10.1007/s10811-006-9094-y

[14] Shibata, T., Fujimoto, K., Nagayama, K., Yamaguchi, K. and Nakamura, T. (2002) Inhibitory Activity of Brown Algal Phlorotannins against Hyaluronidase. International Journal of Food Science and Technology, 37, 703-709. http://dx.doi.org/10.1046/j.1365-2621.2002.00603.x

[15] Shibata, T., Miyasaki, T., Miyake, H., Tanaka, R. and Kawaguchi, S. (2014) The Influence of Phlorotannins and Bromophenols on the Feeding Behavior of Marine Herbivorous Gastropod Turbo cornutus. American Journal of Plant Sciences, 5, 387-392. http://dx.doi.org/10.4236/ajps.2014.53051

[16] Wu, X., Beecher, G.R., Holden, J.M., Haytowitz, D.B., Gebhardt, S.E. and Prior, R.L. (2004) Lipophilic and Hydrophilic Antioxidant Capacities of Common Foods in the United States. Journal of Agricultural and Food Chemistry, 52, 4026-4037. http://dx.doi.org/10.1021/jf049696w

[17] Zhu, Q.Y., Hackman, R.M., Ensunsa, J.L., Holt, R.R. and Keen, C.L. (2002) Antioxidative Activities of Oolong Tea. Journal of Agricultural and Food Chemistry, 50, 6929-6934. http://dx.doi.org/10.1021/jf0206163

[18] Fujii, Y., Tanaka, R., Miyake, H., Tamaru, Y., Ueda, M. and Shibata, T. (2013) Evaluation for Antioxidative Properties of Phlorotannins Isolated from the Brown Alga Eisenia bicyclis, by the H-ORAC Method. Food and Nutrition Sciences, 4, 78-82. http://dx.doi.org/10.4236/fns.2013.48A010

[19] Kellogg, J., Grace, M.H. and Lila, M.A. (2014) Phlorotannins from Alaskan Seaweed Inhibit Carbolytic Enzyme Activity. Marine Drugs, 12, 5277-5294. http://dx.doi.org/10.3390/md12105277

[20] Heffernan, N., Brunton, N.P., FitzGerald, R.J. and Smyth, T.J. (2015) Profiling of the Molecular Weight and Structural Isomer Abundance of Macroalgae-Derived Phlorotannins. Marine Drugs, 13, 509-528. http://dx.doi.org/10.3390/md13010509

[21] Kang, S.M., Lee, S.H., Heo, S.J., Kim, K.N. and Jeon, Y.J. (2011) Evaluation of Antioxidant Properties of a New Compound, Pyrogallol-phloroglucinol-6,6'-bieckol Isolated from Brown Algae, Ecklonia cava. Nutrition Research and Practice, 5, 495-502. http://dx.doi.org/10.4162/nrp.2011.5.6.495

[22] Kang, S.M., Heo, S.J., Kim, K.N., Lee, S.H. and Jeon, Y.J. (2012) Isolation and Identification of New Compound, 2,7' '-phloroglucinol-6,6'-bieckol from Brown Algae, Ecklonia cava and Its Antioxidant Effect. Journal of Functional Foods, 4, 158-166. http://dx.doi.org/doi:10.1016/j.jff.2011.10.001

[23] Yotsu-Yamashita, M., Kondo, S., Segawa, S., Lin, Y.C., Toyohara, H., Ito, H., Konoki, K., Cho, Y. and Uchida, T. (2013) Isolation and Structural Determination of Two Novel Phlorotannins from the Brown Alga Ecklonia kurome Okamura, and Their Radical Scavenging Activities. Marine Drugs, 11, 165-183. http://dx.doi.org/10.3390/md11010165 
[24] Henning, S.M., Fajardo-Lira, C., Lee, H.W., Youssefian, A.A., Go, V.L.W. and Heber, D. (2003) Catechin Content of 18 Teas and a Green Tea Extract Supplement Correlates with the Antioxidant Capacity. Nutrition and Cancer, 45, 226235. http://dx.doi.org/10.1207/S15327914NC4502_13

[25] Parys, S., Kehraus, S., Krick, A., Glombitza, K.W., Carmeli, S., Klimo, K., Gerhäuser, C. and König, G.M. (2010) In Vitro Chemopreventive Potential of Fucophlorethols from the Brown Alga Fucus vesiculosus L. by Anti-Oxidant Activity and Inhibition of Selected Cytochrome P450 Enzymes. Phytochemistry, 71, 221-229.

http://dx.doi.org/doi:10.1016/j.phytochem.2009.10.020

[26] Apostolidis, E., Karayannakidis, P.D., Kwon, Y.I., Lee, C.M. and Seeram, N.P. (2011) Seasonal Variation of Phenolic Antioxidant-Mediated $\alpha$-Glucosidase Inhibition of Ascophyllum nodosum. Plant Foods for Human Nutrition, 66, 313319. http://dx.doi.org/10.1007/s11130-011-0250-4

[27] Wang, T., Jónsdóttir, R. and Ólafsdóttir, G. (2009) Total Phenolic Compounds, Radical Scavenging and Metal Chelation of Extracts from Icelandic Seaweeds. Food Chemistry, 116, 240-248. http://dx.doi.org/10.1016/j.foodchem.2009.02.041

[28] Plaza, M., Amigo-Benavent, M., del Castillo, M.D., Ibáñez, E. and Herrero, M. (2010) Facts about the Formation of New Antioxidants in Natural Samples after Subcritical Water Extraction. Food Research International, 43, 2341-2348. http://dx.doi.org/10.1016/j.foodres.2010.07.036

[29] Balboa, E.M., Conde, E., Moure, A., Falqué, E. and Domínguez, H. (2013) In Vitro Antioxidant Properties of Crude Extracts and Compounds from Brown Algae. Food Chemistry, 138, 1764-1785. http://dx.doi.org/10.1016/j.foodchem.2012.11.026

[30] Ishimoto, H., Tai, A., Yoshimura, M., Amakura, Y., Yoshida, T., Hatano, T. and Ito, H. (2012) Antioxidative Properties of Functional Polyphenols and Their Metabolites Assessed by an ORAC Assay. Bioscience, Biotechnology, and Biochemistry, 76, 395-399. http://dx.doi.org/10.1271/bbb.110717

[31] Fujita, T. (2002) Formation and Removal of Reactive Oxygen Species, Lipid Peroxides and Free Radicals, and Their Biological Effects. YAKUGAKU ZASSHI, 122, 203-218. http://dx.doi.org/10.1248/yakushi.122.203

[32] Nagata, T., Todoriki, S., Masumizu, T., Suda, I., Furuta, S., Du, Z. and Kikuchi, S. (2003) Levels of Active Oxygen Species Are Controlled by Ascorbic Acid and Anthocyanin in Arabidopsis. Journal of Agricultural and Food Chemistry, 51, 2992-2999. http://dx.doi.org/10.1021/jf026179+

[33] Kaneyuki, T., Noda, Y., Traber, M.G., Mori, A. and Packer, L. (1999) Superoxide Anion and Hydroxyl Radical Scavenging Activities of Vegetable Extracts Measured Using Electron Spin Resonance. Biochemistry and Molecular Biology International, 47, 979-989. http://dx.doi.org/10.1080/15216549900202103

[34] Liu, W., Ogata, T., Sato. S., Unoura, K. and Onodera, J. (2001) Superoxide Scavenging Activities of Sixty Chinese Medicines Determined by an ESR Spin-Trapping Method Using Electrogenerated Superoxide. YAKUGAKU ZASSHI, 121, 265-270. http://dx.doi.org/10.1248/yakushi.121.265

[35] Antolovich, M., Prenzler, P.D., Patsalides, E., McDonald, S. and Robards, K. (2002) Methods for Testing Antioxidant Activity. Analyst, 127, 183-198. http://dx.doi.org/10.1039/B009171P

[36] Duan, X.J., Zhang, W.W., Li, X.M. and Wang, B.G. (2006) Evaluation of Antioxidant Property of Extract and Fractions Obtained from a Red Alga, Polysiphonia urceolata. Food Chemistry, 95, 37-43. http://dx.doi.org/10.1016/j.foodchem.2004.12.015

[37] Wang, B.G., Zhang, W.W., Duan, X.J. and Li, X.M. (2009) In Vitro Antioxidative Activities of Extract and SemiPurified Fractions of the Marine Red Alga, Rhodomela confervoides (Rhodomelaceae). Food Chemistry, 113, 11011105. http://dx.doi.org/10.1016/j.foodchem.2008.08.078

[38] Wang, T., Jónsdóttir, R., Liu, H., Gu, L., Kristinsson, H.G., Raghavan, S. and Ólafsdóttir, G. (2012) Antioxidant Capacities of Phlorotannins Extracted from the Brown Algae Fucus vesiculosus. Journal of Agricultural and Food Chemistry, 60, 5874-5883. http://dx.doi.org/10.1021/jf3003653

[39] Shin, H.C., Kim, S.H., Park, Y., Lee, B.H. and Hwang, H.J. (2012) Effects of 12-Week Oral Supplementation of Ecklonia cava Polyphenols on Anthropometric and Blood Lipid Parameters in Overweight Korean Individuals: A DoubleBlind Randomized Clinical Trial. Phytotherapy Research, 26, 363-368. http://dx.doi.org/10.1002/ptr.3559 